\title{
Dec1 expression predicts prognosis and the response to temozolomide chemotherapy in patients with glioma
}

\author{
XIAO-MING LI ${ }^{1 *}$, WEI LIN ${ }^{1 *}$, JIANG WANG $^{1 *}$, WEI ZHANG ${ }^{1}$, AN-AN YIN $^{1}$, YI HUANG $^{2}$, \\ JIAN ZHANG ${ }^{3}$, LIBO YAO $^{3}$, HUAN BIAN $^{4}$, JING ZHANG $^{3}$ and XIANG ZHANG ${ }^{1 *}$ \\ Departments of ${ }^{1}$ Neurosurgery and ${ }^{2}$ Anesthesiology, Xijing Hospital; ${ }^{3}$ Department of Biochemistry and Molecular Biology, \\ School of Basic Medical Science, Center of Teaching Experiment; ${ }^{4}$ Cadet Brigade Team Three, \\ Fourth Military Medical University, Xi'an, Shaanxi 710032, P.R. China
}

Received August 19, 2015; Accepted September 6, 2016

DOI: $10.3892 / \mathrm{mmr} .2016 .5921$

\begin{abstract}
Differentiated embryo chondrocyte expressed gene 1 (Dec1), a crucial cell differentiation mediator and apoptosis inhibitor, is abundantly expressed in various types of human cancer and is associated with malignant tumor progression. As poor differentiation and low apoptosis are closely associated with poor survival rates and a poor response to radio/chemotherapy in patients with cancer, the prognostic value of Decl expression was examined in the present study and its correlation with response to temozolomide (TMZ) chemotherapy was analyzed in patients with glioma. Dec1 expression was analyzed by immunohistochemistry in 157 samples of newly diagnosed glioma and 63 recurrent glioblastoma cases that relapsed during TMZ chemotherapy. Correlations with clinical variables, prognosis and the response to TMZ chemotherapy were analyzed in the newly diagnosed gliomas. Dec1 expression was also compared with the apoptosis index determined by TdT-mediated dUTP nick ending-labeling assay in recurrent glioblastomas. The antiglioma effect of TMZ in nude mice xenografts with Dec1 expression was examined in vivo. High expression of Dec1, which was significantly associated with high pathological tumor grade and poor response to TMZ chemotherapy, was demonstrated to be an unfavorable independent prognostic factor and predicted poor survival in patients with newly diagnosed glioma. In patients with recurrent glioblastoma, there was a negative correlation between Decl expression and the apoptotic index. In nude mice treated with TMZ, Dec1 overexpression potentiated proliferation, but
\end{abstract}

Correspondence to: Professor Wei Lin or Professor Xiang Zhang, Department of Neurosurgery, Xijing Hospital, Fourth Military Medical University, 127 Changle West Road, Xi'an, Shaanxi 710032, P.R. China

E-mail: linwei@fmmu.edu.cn

E-mail: xzhang@fmmu.edu.cn

*Contributed equally

Key words: Dec1, glioma, temozolomide chemotherapy attenuated TMZ-induced apoptosis. In conclusion, Dec1 is a prognostic factor for the clinical outcome and a predictive factor for the response to TMZ chemotherapy in patients with glioma.

\section{Introduction}

Gliomas are the most common primary malignant tumor in the central nervous system and account for $>70 \%$ of all brain tumors (1). The 2007 World Health Organization (WHO) classification criteria distinguishes gliomas into four pathological grades (WHO I to IV) (2). Among them, glioblastoma multiforme (GBM, WHO IV) accounts for approximately half of all gliomas and carries the worst prognosis (3).

Despite the rapid development of multimodal therapeutic strategies over recent years, including surgical resection, local radiotherapy and systemic chemotherapy, the prognosis of gliomas remains dismal. For example, temozolomide (TMZ) is a new generation alkylating agent that has been demonstrated to be one of the most effective chemotherapeutic agents for treating glioma. However, TMZ only extends the overall survival from 12 to 15 months (4), due to intrinsic or acquired chemoresistance to alkylating agents (5). However, the majority of patients with GBM are currently treated with a uniform standardized regimen, regardless of the individual molecular characteristics of each tumor, which may be important for the clinical outcome. Indeed, recent large-scale genomic analyses have demonstrated that remarkable molecular heterogeneity exists in GBMs (6). The molecular information in each tumor tissue is extremely valuable for establishing individualized treatment for gliomas. Thus, the identification of a key molecule, not only for clarifying glioma mechanisms, but also to predict prognosis and the response to treatment in human gliomas has become an increasingly urgent issue.

Differentiated embryo chondrocyte expressed gene 1 (Dec1), also termed split and hairy related protein 2, basic helix-loop-helix binding protein 2 (BHLHB2) or stimulated with retinoic acid 13 (Stra13), is a member of the basic helix-loop-helix (bHLH) family of transcriptional factors. Dec1 has critical functions in various cellular events, including cell differentiation and proliferation (7-9), cell-cycle arrest $(10,11)$, 
inhibition of apoptosis (12-15), immunoregulation (16-18), cellular metabolism (19-21) and circadian rhythms (22-24). Accumulating evidence has identified overexpression of Dec1 in a variety of human tumors and highlighted its contribution to oncogenesis. For example, Stra13 expression was significantly increased during the progression from normal to in situ and invasive breast carcinoma, and positively correlated with the tumor grade (25). Zheng et al (26) identified that Dec1 expression was significantly increased during the tumor progression from well-differentiated to moderately-differentiated and poorly-differentiated gastric cancer tissues (26). Additionally, Stra13 was reported to be abundantly expressed in colon cancers, but not in adjacent normal tissues (12). In renal carcinoma, Stra13 acted as a target of von Hippel-Lindau tumor suppressor protein (pVHL), and its downregulated expression by $\mathrm{pVHL}$ indicated its effect in renal carcinogenesis (27). There was a close association between Dec1 overexpression and hypoxia inducible factor $1-\alpha$, and carbonic anhydrase- 9 in non-small cell lung cancer (28). Together this evidence suggests crucial roles for Dec1 in malignancy progression. However, the profile of Decl expression, or its prognostic and therapeutic significance in human gliomas has not been systematically analyzed.

The present study investigated Dec1 expression by immunohistochemistry (IHC) and further analyzed the correlations between Dec1 expression and clinical variables, prognosis, response to $\mathrm{TMZ}$ chemotherapy and TMZ-induced apoptosis in human glioma specimens. Furthermore, the contribution of Dec1 overexpression to the antiglioma effect of TMZ in vivo was evaluated.

\section{Materials and methods}

Patients and specimens. A total of 157 resected glioma specimens obtained from the Department of Neurosurgery, Xi'Jing Hospital, Fourth Military Medical University (Xi'an, China) from 2007 to 2010, were analyzed in this study. Slides were carefully evaluated and categorized by histological subtype and pathological grade (according to the WHO classifications in 2007) by two pathologists as follows: 45 cases of low-grade glioma (LGG), including grade I (10 pilocytic astrocytomas and 4 myxopapillary ependymomas) and grade II (16 diffuse astrocytomas, 9 oligoastrocytomas and 6 oligodendrogliomas); 112 cases of high-grade glioma (HGG), including grade III (7 anaplastic astrocytomas, 8 anaplastic oligodendrogliomas and 7 anaplastic oligoastrocytomas) and grade IV (90 GBMs). All patients were histologically diagnosed with gliomas, which were disassociated tissues during the initial surgery and did not receive radio- or chemotherapy prior to surgery. Among the newly diagnosed gliomas, 86 patients with HGG were treated with oral TMZ following surgery. The clinical information is presented in Table I. Patients were followed-up for 5 years by telephone or questionnaire letters.

Progression-free survival (PFS) was defined as the time from the date of the initial surgery to the first recurrence (confirmed by MRI) or death. Overall survival (OS) was calculated from the date of the initial surgery until death or the last follow-up. TMZ resistance was standardized as PFS of $>1$ year. Patients who succumbed to diseases not directly associated with glioma or due to unexpected events were excluded from the study. The use of samples in the current study was approved by the Research Ethics Committee of Neurosurgery Department, Xi'Jing Hospital, Fourth Military Medical University (Xi'an, China). Informed consent was obtained from all patients.

IHC and evaluation of staining. IHC assays were performed by the polymer-peroxidase method as follows. Briefly, the $3-\mu \mathrm{m}$ thick slides were deparaffinized and rehydrated, then the sections were rinsed in distilled water for $1 \mathrm{~min}$. Antigen retrieval was performed by boiling in $0.01 \mathrm{M}$ citrate buffer ( $\mathrm{pH}$ 6.0) in a pressure cooker for 86 sec. Following cooling, $0.3 \%$ hydrogen peroxide was used to block nonspecific sites for $30 \mathrm{~min}$, and sections were then incubated with Triton-X for $20 \mathrm{~min}$. Following a wash with PBS and incubation with goat serum (1:20 dilution; OriGene Technologies, Inc.) at room temperature for $1 \mathrm{~h}$, sections were incubated with rabbit polyclonal anti-Dec1 antibody (1:250 dilution; cat. no. ab90594; Abcam, Cambridge, MA, USA) or rabbit monoclonal Ki-67 antibody (1:500-1,000 dilution; cat. no. ab16667; Abcam) at $4^{\circ} \mathrm{C}$ overnight. The specimens were rinsed briefly in PBS and incubated at room temperature first with horseradish peroxidase-conjugated anti-rabbit antibody (cat. no. TA130023; OriGene Technologies, Inc., Beijing, China) for $30 \mathrm{~min}$, and then avidin-biotin peroxidase (OriGene Technologies, Inc.) and $20 \mathrm{~min}$. The slides were stained with diaminobenzidine (OriGene Technologies, Inc.) and water was added to terminate the staining. Slides were counterstained with Meyer's hematoxylin (Baso Diagnostics, Inc., Zhuhai, China).

Dec1 immunostaining was evaluated independently and blindly by two pathologists. The results were compared and re-examined until a consensus was reached for each score. The immunoreactivity score (IRS) of Dec1 was evaluated according to the percentage of positive cells and the staining intensity. IRS was determined by multiplying the two factors: i) Percentage of positive cells $(<5 \%$, scored $0 ; 6-25 \%$, scored $1 ; 26-50 \%$, scored $2 ; 51-75 \%$, scored $3 ;>75 \%$, scored 4 ); and ii) staining intensity (negative staining, 0 ; weak staining, 1; moderate staining, 2; strong staining, 3). The proliferative index was calculated by determining the percentage of KI-67-positive cells.

TdT-mediated dUTP nick ending-labeling (TUNEL) assay. To investigate TMZ-induced apoptosis of tumor cells in glioma patients, an additional 63 patients with recurrent GBM were selected from those that had been histologically documented with malignant glioma and suffered a relapse following the postoperative TMZ chemotherapy. Specimens of these recurrent GBM patients were isolated from the second surgery and used for the TUNEL assay with a DeadEnd ${ }^{\mathrm{TM}}$ Fluorometric TUNEL system (Promega Corporation, Madison, WI, USA), which was performed according the manufacturer's instructions. Tissues were counterstained with DAPI. Images were captured using a fluorescent microscope (Olympus BX51; Olympus Corporation, Tokyo, Japan) using an RGB filter. To evaluate the percentage of apoptotic cells (apoptotic index) in human glioma specimens, TIFF (RGB) images were transferred into the RGB channel in Adobe Photoshop CS5 software (Adobe System Inc., San Jose, CA, USA). Identical 
adjustment of contrast and brightness was performed within for all images presented.

Cell culture, plasmid construction and stable gene transfection. The U87 GBM cell line (American Type Culture Collection, Manassas, VA, USA) was maintained as an adherent monolayer culture in Dulbecco's modified Eagle's medium (Gibco; Thermo Fisher Scientific, Inc., Waltham, MA, USA) supplemented with $10 \%$ fetal bovine serum (Sigma-Aldrich; Merck Millipore, Darmstadt, Germany) in a humidified incubator containing $5 \% \mathrm{CO}_{2}$ at $37^{\circ} \mathrm{C}$.

Plasmids containing full-length Dec1 were generated by reverse transcription-polymerase chain reaction and subcloned into the pENTR ${ }^{\mathrm{TM}} 3 \mathrm{C}$ vector (Invitrogen; Thermo Fisher Scientific, Inc.). Primers for Dec1 were as follows: 5'-ggaagg agttcgaaccatggagcggatccccagcgcgca-3' (forward), and 5'-tgc ggccgcactcgagctagtctttggtttctaagtttaaag-3' (reverse). Dec1 pLenti-6.3 expression vectors were obtained using the Gateway $^{\mathrm{TM}}$ technology (Invitrogen; Thermo Fisher Scientific, Inc.). The pLenti-6.3-cherry vector was used as a negative control. The recombinant expression vector and control vector were packaged in 293T cells (American Type Culture Collection) with the plasmids psPAX2 and pMD2.G (Invitrogen; Thermo Fisher Scientific, Inc.) using Lipofectamine 2000 (Invitrogen; Thermo Fisher Scientific, Inc.) according to the manufacturer's instructions. U87 stable cell lines overexpressing Dec1 and cherry were selected with $8 \mathrm{mg} / \mathrm{ml}$ blasticidin (Invitrogen; Thermo Fisher Scientific, Inc.) and maintained in medium containing $4 \mathrm{mg} / \mathrm{ml}$ blasticidin.

Tumor generation and drug treatment in a mouse model. Nude female BALB/c mice (18-20 g) used for all in vivo studies were obtained from the Laboratory Animal Center of the Fourth Military Medical University (Xi'an, China), and experiments were performed in accordance with guidelines of the Fourth Military Medical University Animal Care and Use Committee. The mice were maintained under pathogen-free conditions in an air flow cabinet at $23^{\circ} \mathrm{C}$, with $12 \mathrm{~h} / 12 \mathrm{~h}$ day/night cycle, and water and food ad libitum. When mice were six weeks old, tumor generation was initiated by subcutaneous injection of $1 \times 10^{6}$ stable U87 cells overexpressing Dec1 or cherry $(n=5$ per group) in each flank of a nude mouse. Palpable tumors were generated $\sim 5-7$ days postinoculation, and TMZ was added to the diet at $50 \mathrm{mg} / \mathrm{kg}$ for 5 consecutive days. The tumor size was measured with a slide caliper and the tumor volume was recorded using the following formula: Volume $=a \mathrm{x} \mathrm{b}^{2} / 2$ ( $a=$ the larger dimension; $b=$ the smaller dimension). Following 30 days of inoculation, the mice were sacrificed by decapitation. Tumors were removed, weighed and paraffin-embedded. Pathological sections $(3-\mu \mathrm{m})$ were prepared for TUNEL and Ki-67 immunostaining analysis.

Statistical analysis. All statistical analyses were performed using the SPSS 13.0 package (SPSS Inc., Chicago, IL, USA). Values are presented as the mean \pm standard deviation. Relationships between Decl expression levels and clinical variables were analyzed by the Mann-Whitney test or the Kruskal-Wallis test. The Kaplan-Meier method was used to plot survival curves and the prognostic differences in groups with different Dec1 expression were tested by log-rank analysis. Hazard ratios
(HR) of different prognostic factors were evaluated by Cox multivariate analysis. Differences of Decl IRS and the apoptotic index (AI) between the low Decl expression group and the high Dec1 expression group or between the TMZ-resistant group and the TMZ-sensitive group were compared using the Student's $t$-test. The nonparametric Spearman's rank correlation coefficient was performed to assess the correlation between AI and Dec1 IRS. $\mathrm{P}<0.05$ was considered to indicate a statistically significant difference.

\section{Results}

Correlation between Decl expression with clinical variables in glioma patients. Correlations between Dec1 expression levels with clinical variables were analyzed in 157 cases of newly diagnosed gliomas (Table I). Dec1 expression levels were evaluated by IHC, and patients were stratified as low Dec1 expression (IRS $<8$ ) or high Decl expression (IRS $\geq 8$ ), according to the mean IRS of Dec1 (6.44 3 3.73). Among the total 157 cases, 82 patients exhibited low Dec1 expression, whereas 75 cases exhibited high Dec1 expression. Representative staining images are presented in Fig. 1. In LGG, a lower number of Dec1 positive cells was observed exhibiting a weak staining intensity, whereas in HGG, nearly all tumor cells were strongly stained. Decl expression increased with the progression of pathologic grades $(\mathrm{P}=0.023)$. In addition, Dec1 expression was also significantly correlated with the Karnofsky performance status (KPS; $\mathrm{P}=0.016$ ). No statistically significance was detected between the expression levels of Dec1 and age, gender, tumor location, tumor size or surgical resection.

High Decl expression is associated with poor prognosis in patients with glioma. The Kaplan-Meier analyses for PFS and OS were performed to evaluate the prognostic differences between Dec1 expression groups in patients with glioma. The postoperative mean PFS and OS of all eligible patients with glioma were 18.1 and 23.1 months, respectively. The postoperative mean PFS and OS of patients with low Dec1 expression were 22 and 27 months, respectively, whereas those of patients with high Decl expression were 13.8 and 18.8 months, respectively. Patients with low Decl expression exhibited longer PFS and OS compared to those with high Decl expression (log-rank test, $\mathrm{P}=0.0001$ for both; Fig. 2A). In addition, the considered variables, including age, KPS and WHO grade, also exhibited statistically significance at log-rank test in all cases of glioma for PFS and OS (data not shown). However, gender, tumor location, tumor size or surgery resection had no prognostic value for PFS and OS in the present cohort (data not shown).

Kaplan-Meier analyses were also performed in different subgroups stratified according to the malignant degree. As high pathological grade of gliomas is considered a marker of high risk, the patients were classified into two subgroups: LGG and HGG. In LGG, the postoperative mean PFS and OS were 34.5 and 42 months, respectively. The postoperative mean PFS and OS of patients with low Dec1 expression were 39.2 and 45.3 months, respectively, whereas those of patients with high Dec1 expression were 26.8 and 36.6 months, respectively. High Decl expression was significantly correlated with shorter PFS and OS in LGG (log-rank test, $\mathrm{P}=0.0085$ and $\mathrm{P}=0.0135$, respectively; Fig. 2B). Similarly in $\mathrm{HGG}$, the postoperative 
Table I. Correlations between Decl expression and the clinical variables in glioma patients.

\begin{tabular}{|c|c|c|c|c|c|}
\hline Variable & Description & $\mathrm{n}$ & $\begin{array}{l}\text { Dec1-low } \\
\text { expression }\end{array}$ & $\begin{array}{l}\text { Dec1-high } \\
\text { expression }\end{array}$ & P-value \\
\hline \multirow[t]{2}{*}{ Age (years) } & $<50$ & 76 & 36 & 40 & \multirow[t]{2}{*}{$0.239^{\mathrm{a}}$} \\
\hline & $\geq 50$ & 81 & 46 & 35 & \\
\hline \multirow[t]{2}{*}{ Gender } & Male & 75 & 45 & 30 & \multirow[t]{2}{*}{$0.063^{\mathrm{a}}$} \\
\hline & Female & 82 & 37 & 45 & \\
\hline \multirow[t]{2}{*}{ KPS } & $<80$ & 56 & 22 & 34 & \multirow[t]{2}{*}{$0.016^{\mathrm{a}}$} \\
\hline & $\geq 80$ & 101 & 60 & 41 & \\
\hline \multirow[t]{4}{*}{ Grade } & I & 14 & 12 & 2 & \multirow[t]{4}{*}{$0.023^{b}$} \\
\hline & II & 31 & 16 & 15 & \\
\hline & III & 22 & 14 & 8 & \\
\hline & IV & 90 & 40 & 50 & \\
\hline \multirow[t]{2}{*}{ Tumor location } & Supratentorial & 94 & 50 & 44 & \multirow[t]{2}{*}{$0.769^{\mathrm{a}}$} \\
\hline & Infratentorial & 63 & 32 & 31 & \\
\hline \multirow[t]{2}{*}{ Tumor size } & $<3 \mathrm{~cm}$ & 78 & 38 & 40 & \multirow[t]{2}{*}{$0.383^{\mathrm{a}}$} \\
\hline & $\geq 3 \mathrm{~cm}$ & 79 & 44 & 35 & \\
\hline \multirow[t]{2}{*}{ Surgery resection $^{\mathrm{c}}$} & Total & 91 & 48 & 43 & \multirow[t]{2}{*}{$0.879^{\mathrm{a}}$} \\
\hline & Subtotal & 66 & 34 & 32 & \\
\hline \multirow[t]{2}{*}{ TMZ chemotherapy ${ }^{\mathrm{d}}$} & Resistant & 46 & 16 & 30 & \multirow[t]{2}{*}{$0.020^{\mathrm{a}}$} \\
\hline & Sensitive & 40 & 24 & 16 & \\
\hline
\end{tabular}

P-values were estimated using the ${ }^{a}$ Mann-Whitney test and the ${ }^{b}$ Kruskal-Wallis test. ${ }^{c}$ Surgery resection was evaluated by postoperative MRI within $72 \mathrm{~h}$ after operation. Complete resolution on the postoperative MRI (T1 low-intensity lesion for grade I, II and III; T1 gadolinium-enhanced lesion for grade IV) was considered as total resection. ${ }^{\mathrm{d}} \mathrm{TMZ}$ chemotherapy; 86 cases of high-grade glioma patients were treated with oral TMZ after surgery. TMZ resistance was standardized as the progression-free survival of $<1$ year. Dec1, differentiated embryo chondrocyte expressed gene 1; MRI, magnetic resonance imaging; TMZ, temozolomide.



Figure 1. Representative images of differentiated embryo chondrocyte expressed gene 1 immunostaining in human glioma tissues. (A) Pilocytic astrocytoma, WHO I; (B) astrocytoma, WHO II; (C) anaplastic astrocytoma, WHO III; and (D) glioblastoma, WHO IV. WHO, World Health Organization grade.

mean PFS and OS were 11.5 and 15.5 months, respectively. The postoperative mean PFS and OS of patients with low
Dec1 expression were 13.1 and 17.6 months, respectively, while those of patients with high Decl expression were 10 and 13.6 months, respectively. Dec1 expression in $\mathrm{HGG}$ also proved to be a prognostic factor for both PFS and OS (log-rank test, $\mathrm{P}=0.0175$ and $\mathrm{P}=0.0070$, respectively; Fig. $2 \mathrm{C}$ ). In addition, age and KPS had prognostic value for PFS and OS in LGG, while radiotherapy postsurgery did not contribute to long-term benefits in the current cohort (data not shown). In HGG, age, KPS and standard treatment also significantly predicted PFS and OS (data not shown).

Using the Cox proportional hazards model, univariate and multivariate analyses were performed in patients with glioma (Tables II and III). In LGG, low Dec1 expression was identified as a favorable prognostic indicator for PFS $(\mathrm{P}=0.009$; HR, 0.226) and $\mathrm{OS}(\mathrm{P}=0.010 ; \mathrm{HR}, 0.246)$. Similarly, age and KPS were also identified as independent prognostic factors for PFS and OS. However, for PFS and OS, no statistically significance was found among other variables, including gender, tumor location, tumor size, surgery resection or radiotherapy (Table II). The Cox proportional hazards model in HGG also revealed low Decl expression as a favorable predictor of $\mathrm{PFS}(\mathrm{P}=0.015$; $\mathrm{HR}, 0.581)$ and $\mathrm{OS}(\mathrm{P}=0.009 ; \mathrm{HR}, 0.569)$. In addition, KPS and standard treatment were also demonstrated to be independent prognostic factors for PFS and OS in HGG, whereas other variables, including age, gender, tumor location, tumor size and surgery resection were not prognostic factors (Table III). 

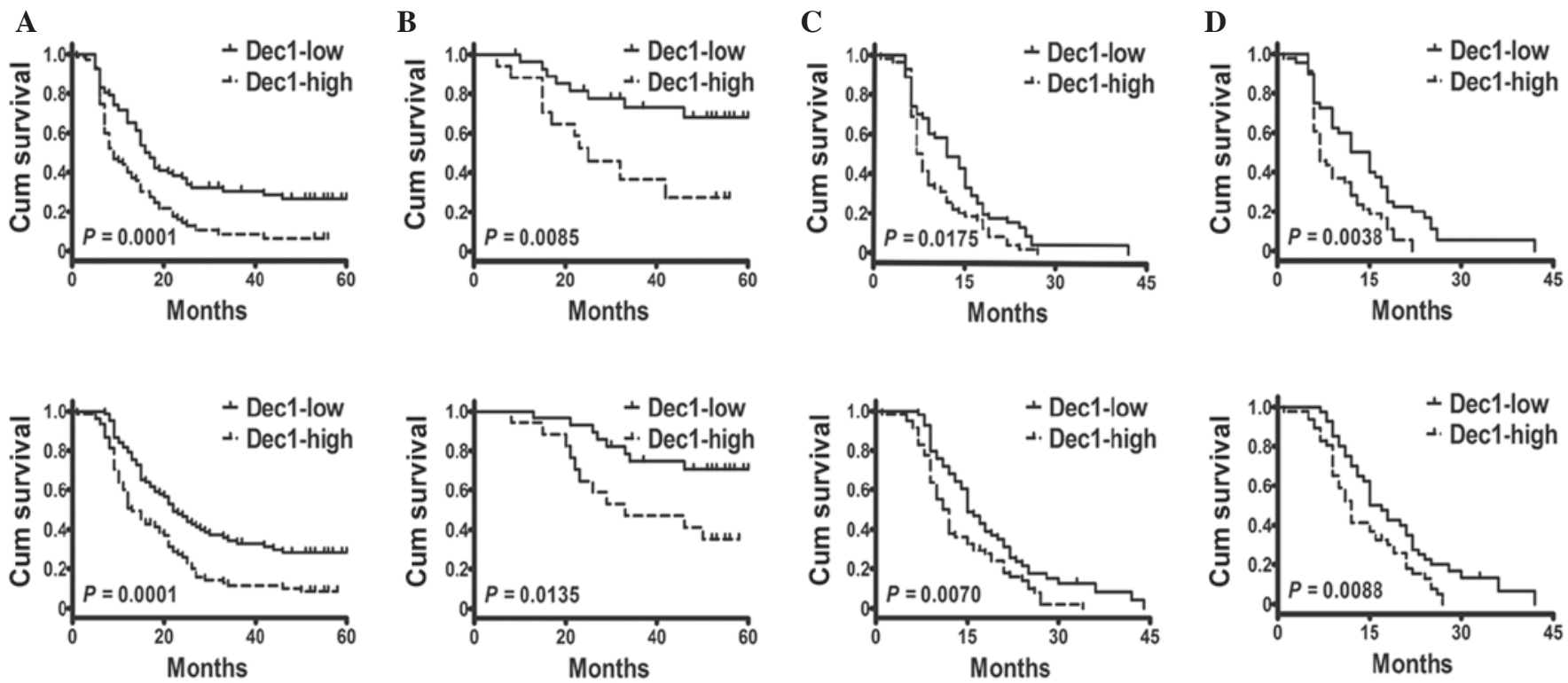

Figure 2. Correlation of Dec1 expression with progression-free survival (upper) and overall survival (lower) in (A) all cases, (B) low-grade gliomas, (C) high-grade gliomas and (D) temozolomide-treated gliomas. Cum, cumulative; Dec1, differentiated embryo chondrocyte expressed gene 1.

High Decl expression predicts poor responses to TMZ chemotherapy in $H G G$ patients. Among the patients with HGG, 86 cases were treated with TMZ following surgery. The mean Dec1 IRS in all TMZ-treated patients with HGG was $7.14 \pm 3.87$, and the mean Dec1 IRS in the TMZ-resistant group $(8.39 \pm 3.44)$ was significantly higher than that of the TMZ-sensitive group $(5.7 \pm 3.86, \mathrm{t}=3.389 ; \mathrm{P}=0.001)$. In addition, 30 out of $46(65.2 \%)$ patients with high Dec1 expression exhibited resistance to TMZ chemotherapy, compared with 16 out of $40(40 \%)$ patients with low Dec1 expression. A statistically significant correlation was observed between Dec1 expression level and the response to TMZ chemotherapy $(\mathrm{P}=0.020$; Table I). The mean postoperative PFS and OS of all TMZ-treated patients with HGG were 11.9 and 15.9 months, respectively. The mean PFS and OS of patients with low Dec1 expression were 14.3 and 18.3 months, respectively, whereas those of patients with high Decl expression were 9.7 and 13.7 months, respectively. HGG patients with low Dec1 expression exhibited better survival than those with high Dec1 expression when they received TMZ chemotherapy (log-rank test, $\mathrm{P}=0.0038$ and $\mathrm{P}=0.0088$, respectively; Fig. 2D).

Correlation of Decl expression with apoptosis in TMZ-treated recurrent GBMs. All 63 cases of TMZ-treated recurrent GBM specimens were examined by Dec1 IHC and the TUNEL assay, and representative images of both are presented in Fig. 3A. TMZ-induced apoptotic cells were detected in all these specimens. The AI was expressed as the percentage of the apoptotic cells and ranged from $0.31-5.81 \%$ (mean, $2.18 \pm 1.35 \%$ ). The AI in low Dec1 expression group $(2.70 \pm 1.42 \%)$ was significantly higher compared with the high Decl expression group $(1.76 \pm 1.15 \%$; $t=2.822, \mathrm{P}=0.007$; Fig. 3B). Furthermore, the increase of Dec1 IRS in the recurrent GBM specimens was significantly correlated with a decrease of AI ( $r=-0.341$, $\mathrm{P}=0.006$; Fig. $3 \mathrm{C})$. In addition, a significant difference in AI was observed between the TMZ-resistant group and
TMZ-sensitive group $(1.81 \pm 1.20 \%$ and $2.67 \pm 1.40 \%$, respectively; t=-2.539, P=0.014; Fig. 3D).

Decl overexpression significantly promotes the tumorigenicity of U87 cells xenografts in TMZ-treated nude mice. To confirm the correlation between overexpression of Dec1 and TMZ resistance in patients with GBM, the effects of Dec1 overexpression on TMZ resistance were investigated in vivo. U87 GBM cells stably expressing Decl or cherry (control) were established and transplanted into nude mice to generate tumor xenograft models. At $\sim 30$ days post-implantation, tumors were removed. A significant TMZ-resistant effect was observed in the U87-Dec1 tumors compared with the U87-cherry tumors (Fig. 4A). Following treatment with TMZ, the mean tumor volume and weight in U87-Dec1 tumors $\left(1257 \pm 382.7 \mathrm{~mm}^{3}\right.$ and $114 \pm 12.99 \mathrm{mg}$, respectively) was significantly higher compared with the U87-cherry tumors $\left(468.8 \pm 193.4 \mathrm{~mm}^{3}\right.$ and $44 \pm 2.63 \mathrm{mg}$, respectively; $\mathrm{P}<0.001$ for both; Fig. 4B and C). In U87-Dec1 and U87-cherry tumors treated with TMZ, Dec1 levels were assessed by IHC and proliferation and apoptosis was detected by Ki-67 immunostaining and TUNEL, respectively (Fig. 4D). The proliferative index in U87-Dec1 tumors $(51.6 \pm 3.74 \%)$ was significantly higher compared with the U87-cherry tumors $(38.20 \pm 3.55 \%$; $\mathrm{t}=2.600, \mathrm{P}=0.032$; Fig. 4E), and the AI was significantly lower in U87-Dec1 tumors $(15.26 \pm 2.20 \%)$ compared with U87-cherry tumors (44.10 $\pm 2.69 \%$; $\mathrm{t}=8.296, \mathrm{P}<0.0001$; Fig. $4 \mathrm{~F})$.

\section{Discussion}

The present study investigated the expression status of Dec1 in 157 cases of newly diagnosed glioma and 63 cases of TMZ-treated recurrent GBM. High Dec1 expression, which was closely associated with pathological grade, KPS and poor response to TMZ chemotherapy, was a significant risk factor for PFS and OS in the patients with newly diagnosed glioma. In addition, a negative correlation between Decl expression 
Table II. Univariate and multivariate analyses of progression-free survival and overall survival in low-grade glioma.

\begin{tabular}{|c|c|c|c|c|}
\hline Clinical variables & $\begin{array}{l}\text { Unadjusted HR } \mathrm{HR}^{\mathrm{a}} \\
\quad(95 \% \mathrm{CI})\end{array}$ & P-value & $\begin{array}{l}\text { Adjusted HR }{ }^{\mathrm{b}} \\
(95 \% \mathrm{CI})\end{array}$ & P-value \\
\hline \multicolumn{5}{|c|}{ Progression-free survival } \\
\hline Age & & & & \\
\hline$<50$ vs. $\geq 50$ & $0.363(0.141-0.930)$ & 0.035 & $0.273(0.089-0.836)$ & 0.023 \\
\hline Gender & & & & \\
\hline Male vs. female & $1.329(0.534-3.307)$ & 0.540 & $1.350(0.490-3.720)$ & 0.562 \\
\hline $\begin{array}{l}\text { KPS } \\
\geq 80 \text { vs. }<80\end{array}$ & $0.198(0.076-0.513)$ & 0.001 & $0.207(0.059-0.725)$ & 0.014 \\
\hline $\begin{array}{l}\text { Dec1 expression } \\
\text { Low vs. high }\end{array}$ & $0.312(0.124-0.786)$ & 0.013 & $0.226(0.074-0.693)$ & 0.009 \\
\hline $\begin{array}{l}\text { Tumor location } \\
\text { Sup. vs. inf. }\end{array}$ & $1.451(0.550-3.830)$ & 0.452 & $0.720(0.237-2.186)$ & 0.562 \\
\hline $\begin{array}{l}\text { Tumor size } \\
\geq 3 \mathrm{~cm} \text { vs. }<3 \mathrm{~cm}\end{array}$ & $2.814(1.105-7.165)$ & 0.030 & $1.409(0.423-4.691)$ & 0.576 \\
\hline $\begin{array}{l}\text { Surgery resection } \\
\text { Total vs. subtotal }\end{array}$ & $0.820(0.311-2.159)$ & 0.688 & $0.435(0.101-1.874)$ & 0.264 \\
\hline $\begin{array}{l}\text { Radiotherapy } \\
\text { No vs. yes }\end{array}$ & $1.213(0.353-4.165)$ & 0.759 & $2.418(0.388-15.06)$ & 0.344 \\
\hline Overall survival & & & & \\
\hline $\begin{array}{l}\text { Age } \\
<50 \text { vs. } \geq 50\end{array}$ & $0.362(0.141-0.930)$ & 0.035 & $0.283(0.092-0.873)$ & 0.028 \\
\hline $\begin{array}{l}\text { Gender } \\
\text { Male vs. female }\end{array}$ & $1.425(0.570-3.559)$ & 0.448 & $1.055(0.385-2.889)$ & 0.917 \\
\hline $\begin{array}{l}\text { KPS } \\
\geq 80 \text { vs. }<80\end{array}$ & $0.242(0.095-0.618)$ & 0.003 & $0.257(0.080-0.828)$ & 0.023 \\
\hline $\begin{array}{l}\text { Dec1 expression } \\
\text { Low vs. high }\end{array}$ & $0.337(0.135-0.840)$ & 0.020 & $0.246(0.085-0.710)$ & 0.010 \\
\hline $\begin{array}{l}\text { Tumor location } \\
\text { Sup. vs. inf. }\end{array}$ & $1.318(0.501-3.468)$ & 0.576 & $0.585(0.196-1.748)$ & 0.337 \\
\hline $\begin{array}{l}\text { Tumor size } \\
\geq 3 \mathrm{~cm} \text { vs. }<3 \mathrm{~cm}\end{array}$ & $3.007(1.178-7.678)$ & 0.021 & $1.772(0.554-5.664)$ & 0.334 \\
\hline $\begin{array}{l}\text { Surgery resection } \\
\text { Total vs. subtotal }\end{array}$ & $0.890(0.337-2.350)$ & 0.814 & $0.521(0.120-2.266)$ & 0.385 \\
\hline $\begin{array}{l}\text { Radiotherapy } \\
\text { No vs. yes }\end{array}$ & $1.309(0.381-4.501)$ & 0.669 & $1.996(0.312-12.77)$ & 0.466 \\
\hline
\end{tabular}

${ }^{a} \mathrm{HR}$ in univariate models. ${ }^{\mathrm{b}} \mathrm{HR}$ in multivariable models. HR, hazard ratio; KPS, Karnofsky performance status; Dec1, differentiated embryo chondrocyte expressed gene 1; sup, supratentorial; inf, infratentorial.

with the AI was detected in the TMZ-treated recurrent GBMs. In in vivo experiments, Decl overexpression promoted the tumorigenicity of U87 xenografts in nude mice treated with TMZ and significantly attenuated the antiglioma effect of TMZ. Together, the results demonstrated that Dec1 may be a valuable prognostic indicator for clinical outcome, and notably, a predictive factor for the response to TMZ chemotherapy in patients with glioma.

Various studies have investigated Decl to clarify its role in oncogenesis in multiple types of human tumor (12,14,25-30).
However, its value as a prognostic marker has not been previously determined. In the current study, in LGG and HGG, high Dec1 expression was correlated with unfavorable outcomes and was identified as an independent prognostic factor (Fig. 2; Tables II and III). In gliomas, complete resection is nearly impossible for the primary tumor due to its infiltrative nature. Therefore, radiotherapy- or chemotherapy-induced apoptosis of the residual glioma cells will dramatically impact the clinical outcome (Fig. 3D). The anti-apoptotic effect of Dec1 has been previously confirmed by other 
Table III. Univariate and multivariate analyses of progression-free survival and overall survival in high-grade glioma.

\begin{tabular}{|c|c|c|c|c|}
\hline Clinical variables & $\begin{array}{l}\text { Unadjusted HR } \mathrm{HR}^{\mathrm{a}} \\
\quad(95 \% \mathrm{CI})\end{array}$ & P-value & $\begin{array}{l}\text { Adjusted } \mathrm{HR}^{\mathrm{b}} \\
\quad(95 \% \mathrm{CI})\end{array}$ & P-value \\
\hline \multicolumn{5}{|c|}{ Progression-free survival } \\
\hline \multicolumn{5}{|l|}{ Age } \\
\hline$<50$ vs. $\geq 50$ & $0.628(0.420-0.938)$ & 0.023 & $0.768(0.493-1.196)$ & 0.243 \\
\hline \multicolumn{5}{|l|}{ Gender } \\
\hline Male vs. female & $0.860(0.584-1.267)$ & 0.445 & $0.945(0.620-1.440)$ & 0.793 \\
\hline \multicolumn{5}{|l|}{ KPS } \\
\hline$\geq 80$ vs. $<80$ & $0.363(0.234-0.562)$ & 0.000 & $0.570(0.351-0.925)$ & 0.023 \\
\hline \multicolumn{5}{|l|}{ Dec1 expression } \\
\hline Low vs. high & $0.643(0.434-0.954)$ & 0.028 & $0.581(0.375-0.900)$ & 0.015 \\
\hline \multicolumn{5}{|l|}{ Tumor location } \\
\hline Sup. vs. inf. & $0.941(0.631-1.401)$ & 0.763 & $1.052(0.697-1.587)$ & 0.809 \\
\hline \multicolumn{5}{|l|}{ Tumor size } \\
\hline$\geq 3 \mathrm{~cm}$ vs. $<3 \mathrm{~cm}$ & $0.879(0.594-1.299)$ & 0.516 & $0.994(0.643-1.537)$ & 0.978 \\
\hline \multicolumn{5}{|l|}{ Surgery resection } \\
\hline Total vs. subtotal & 1.055 (0.711-1.565) & 0.790 & $0.849(0.564-1.277)$ & 0.432 \\
\hline \multicolumn{5}{|l|}{ Standard treatment ${ }^{c}$} \\
\hline No vs. yes & $3.081(2.031-4.673)$ & 0.000 & $2.823(1.736-4.591)$ & 0.000 \\
\hline \multicolumn{5}{|l|}{ Overall survival } \\
\hline \multicolumn{5}{|l|}{ Age } \\
\hline$<50$ vs. $\geq 50$ & $0.641(0.429-0.956)$ & 0.029 & $0.861(0.554-1.339)$ & 0.506 \\
\hline \multicolumn{5}{|l|}{ Gender } \\
\hline Male vs. female & $0.827(0.561-1.218)$ & 0.336 & $0.877(0.577-1.334)$ & 0.541 \\
\hline \multicolumn{5}{|l|}{ KPS } \\
\hline$\geq 80$ vs. $<80$ & $0.347(0.226-0.535)$ & 0.000 & $0.549(0.340-0.887)$ & 0.014 \\
\hline \multicolumn{5}{|l|}{ Dec1 expression } \\
\hline Low vs. high & $0.598(0.402-0.889)$ & 0.011 & $0.569(0.374-0.866)$ & 0.009 \\
\hline \multicolumn{5}{|l|}{ Tumor location } \\
\hline Sup. vs. inf. & $0.849(0.570-1.266)$ & 0.422 & $0.761(0.501-1.157)$ & 0.202 \\
\hline \multicolumn{5}{|l|}{ Tumor size } \\
\hline$\geq 3 \mathrm{~cm}$ vs. $<3 \mathrm{~cm}$ & $0.815(0.552-1.205)$ & 0.306 & $0.978(0.634-1.509)$ & 0.920 \\
\hline \multicolumn{5}{|l|}{ Surgery resection } \\
\hline Total vs. subtotal & 1.169 (0.788-1.734) & 0.437 & $0.974(0.651-1.455)$ & 0.896 \\
\hline \multicolumn{5}{|l|}{ Standard treatment ${ }^{\mathrm{c}}$} \\
\hline No vs. yes & $3.531(2.340-5.329)$ & 0.000 & 3.124 (1.941-5.027) & 0.000 \\
\hline
\end{tabular}

studies (12-14,31-33). Indeed the results of the present study indicated that Dec1 overexpression significantly decreased apoptosis in vivo (Figs. 3 and 4). Based on this evidence, we speculate that the correlation between high Dec1 expression and the poor outcome may be explained by the ability of Dec1 to confer partial resistance to chemotherapy-induced apoptosis. In addition, HGGs were either poorly differentiated or undifferentiated, and consequently carried a dismal prognosis. The current study observed that Decl expression was increased with increasing WHO grade, which was consistent with its capacity to antagonize differentiation in various types of human tumor $(25,26)$. Furthermore, several previous studies provided evidence of Dec1 involvement in multiple cellular pathways, including p53, VHL/hypoxia and Janus kinase/Signal Transducer and Activator of Transcription signaling, which are crucial for tumor malignancy $(27,31,32,34-36)$. Poor differentiation and high malignancy were closely associated with unfavorable clinical 
A

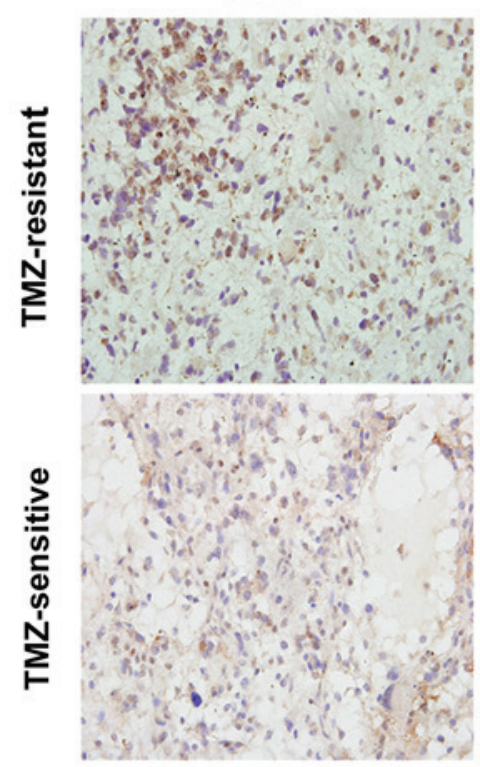

B

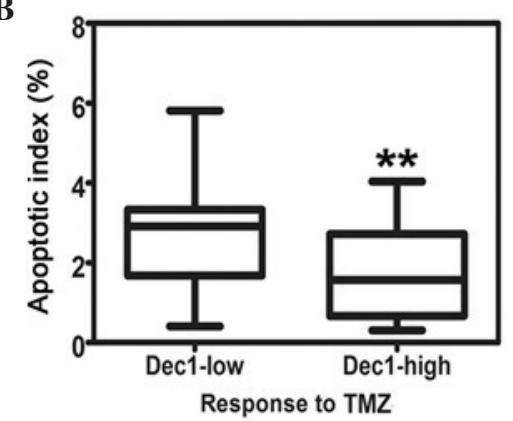

DAPI
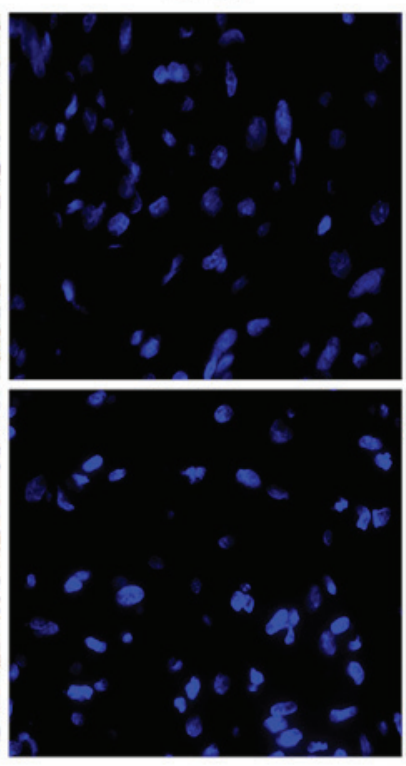

C

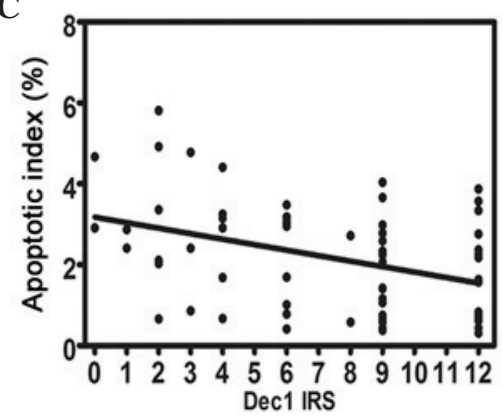

TUNEL
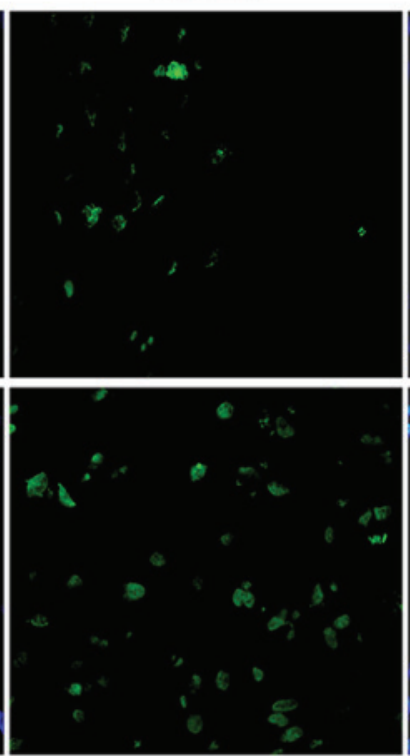

Merge

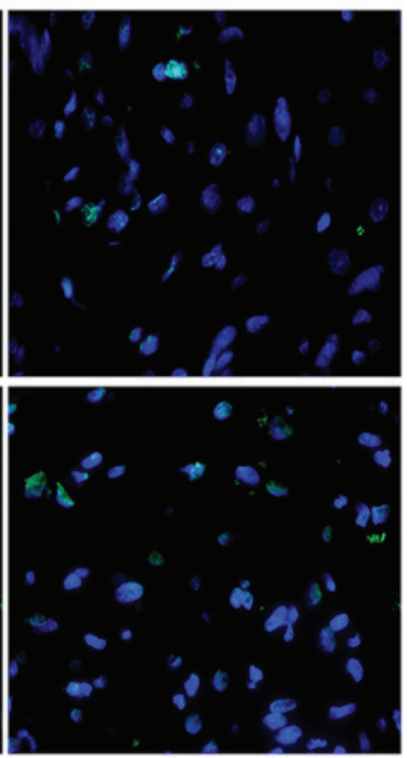

D

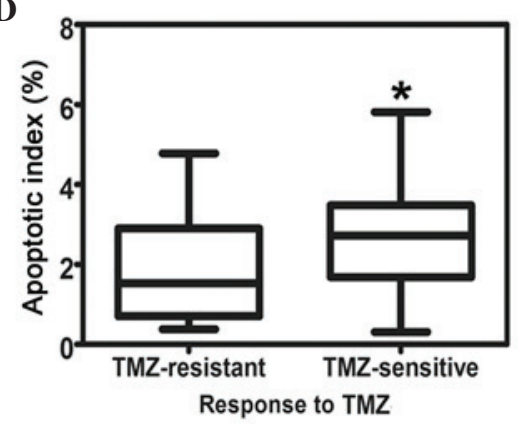

Figure 3. Correlation of Dec1 expression with TMZ-induced apoptosis in TMZ-treated patients with recurrent GBMs. (A) Representative photographs of Dec1 immunostaining (magnification, $\mathrm{x} 400$ ) and apoptosis detection by TUNEL assay (magnification, x1,000). Upper row: recurrent GBM patients resistant to TMZ chemotherapy exhibited high expression of Dec1 and fewer apoptotic tumor cells. Lower row: recurrent GBM patients sensitive to TMZ chemotherapy exhibited low expression of Dec1 and more apoptotic tumor cells. Arrowheads indicate apoptotic cells in recurrent GBM tissues. (B) Box-whisker plot presenting the correlation of Dec1 expression levels with apoptotic index (** $\mathrm{P}=0.006 \mathrm{vs}$. Dec-low). (C) Scatterplot of the correlation of Dec1 IRS with apoptotic index. With the increase in Dec1 IRS, the apoptotic index decreased markedly ( $\mathrm{r}=-0.341)$. By linear regression, a trend line representing the best fit indicated on the scatterplot. (D) Box-whisker plot presenting the correlation of the response to TMZ chemotherapy with apoptotic index ( $\mathrm{P}<0.05$ vs. TMZ-resistant). The values represent the mean \pm standard deviation of 63 fields. GBM, glioblastoma multiforme; Dec1, differentiated embryo chondrocyte expressed gene 1; TUNEL, terminal deoxynucleotidyl transferase dUTP nick end labeling; TMZ, temozolomide; IRS, immunoreactivity score.

outcome. Other reasons for the poor prognosis of gliomas may be the functions of Dec1 in cell differentiation and malignancy. Therefore, inhibition of Dec1 expression may contribute to the long-term benefits for patients with glioma, suggesting that Dec1 might be a novel potential molecular target for glioma biological therapy.

By contrast with the role of Dec1 as a significant risk factor for the poor outcome of gliomas described above, an opposing prognostic effect of Dec1 had been previously reported (37), demonstrating that low BHLHB2 (Dec1) expression predicted shorter survival in pancreatic ductal adenocarcinoma. Notably, the correlation of Decl expression levels with differentiation or malignant progression in various types of human cancer is not unique. In a previous study, Decl expression was increased during the progression from well differentiated to poorly differentiated tumor tissues in gastric cancer, which was consistent with findings in breast cancer and the present study $(25,26)$. Shi et al (30) reported that low Decl expression was associated with poor histological differentiation and malignant progression in hepatocellular carcinoma. These findings indicate that the expression of Dec1 might be a context-dependent prognostic factor. Similar to Dec1, Kruppel like factor 5 (KLF5) has also been previously reported to exert opposing prognostic roles in different types of cancer. For instance, patients with non-small cell lung cancer with high levels of KLF5 expression had a significantly better disease-specific survival compared with intermediate to no KLF5 expression (38). Patients with nuclear KLF5 staining had a significantly lower disease-free survival rate compared with patients with negative nuclear staining in gastric cancer (39). Clarifying the different prognostic effects of Dec1 in various types of human tumor warrants further investigation.

To date, the most important breakthrough and most commonly used strategy in HGG chemotherapy was the introduction of the alkylating agent, TMZ (40). Thus, the response to TMZ chemotherapy significantly affects the prognosis of 
A

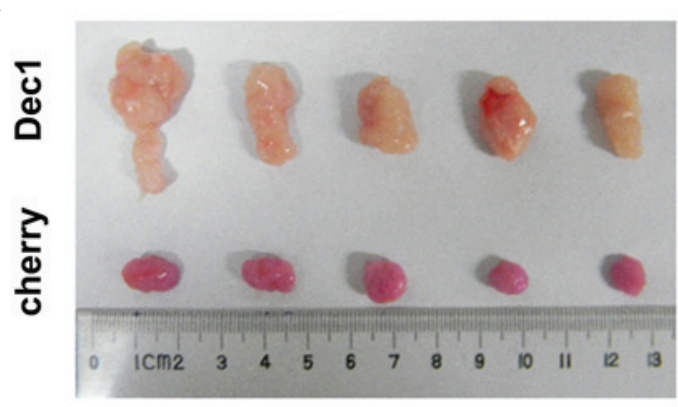

B

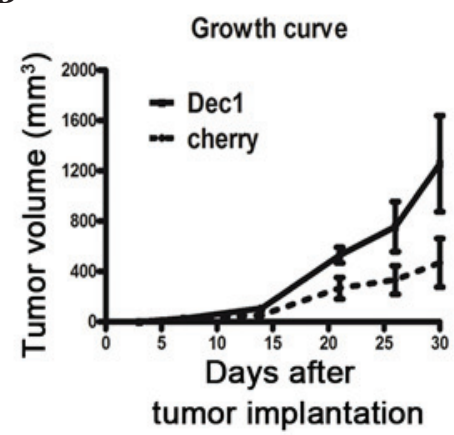

C

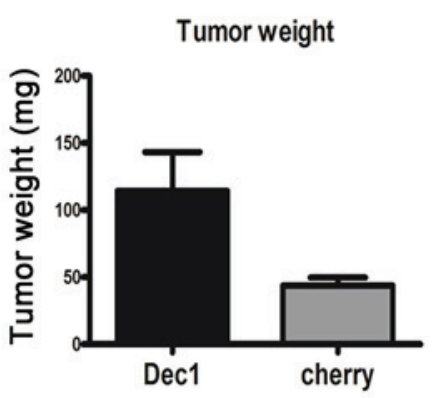

D
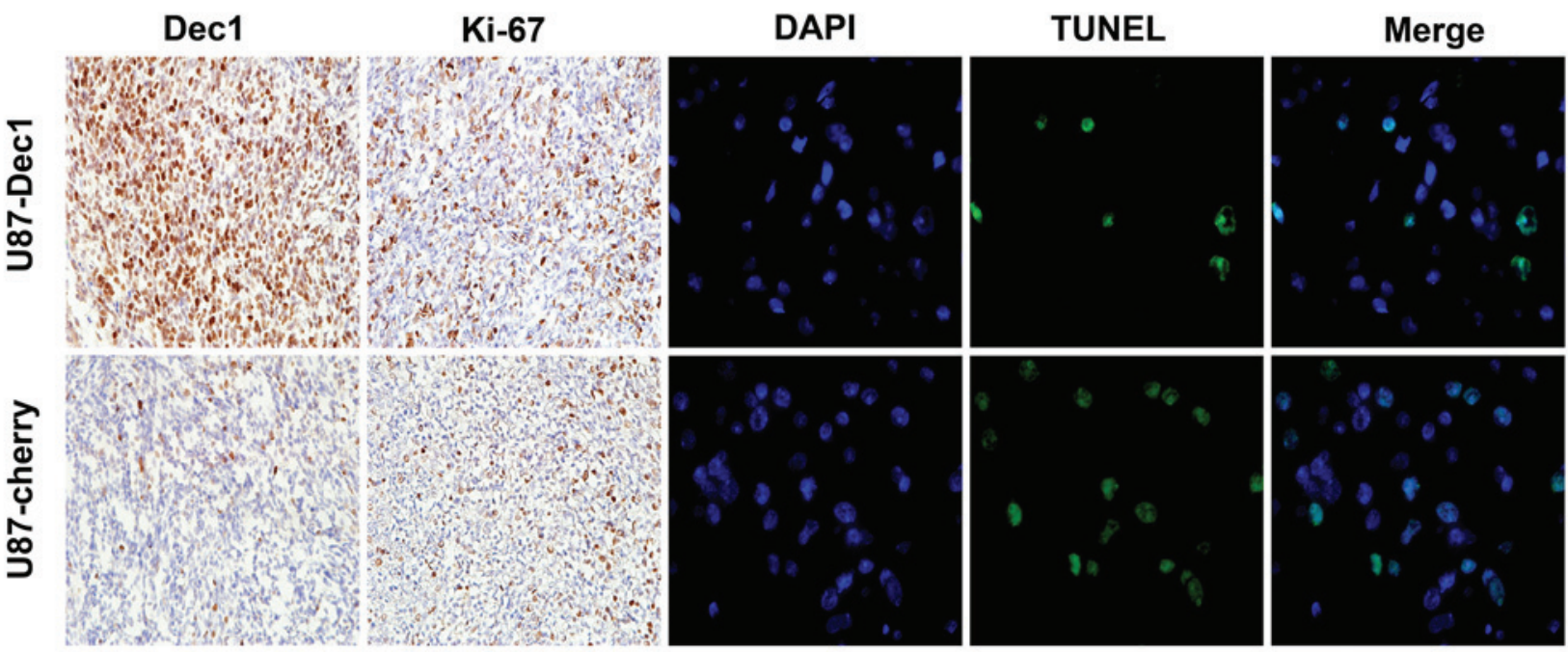

E

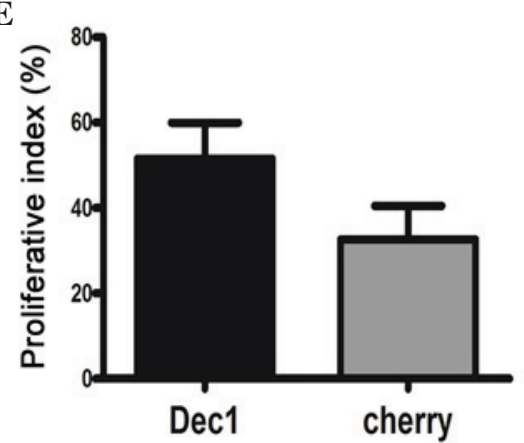

F

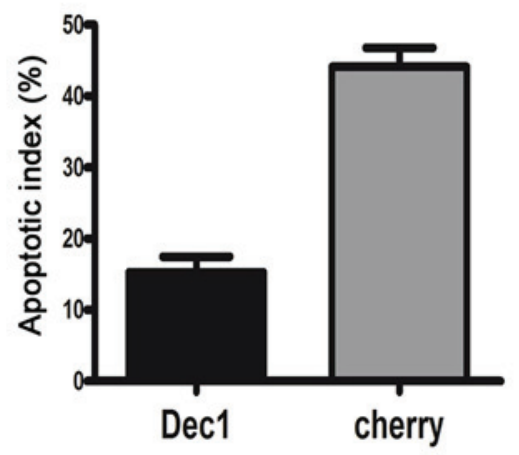

Figure 4. U87 glioblastoma multiforme cells with stable Dec1 expression exhibit a significant TMZ-resistance effect in xenografts of nude mice treated with TMZ. (A) In vivo study: U87-Dec1 and U87-cherry glioma xenografts were generated ( $\mathrm{n}=5)$. After xenografts reached a palpable size, mice were treated with TMZ for 30 days, and glioma xenografts were weighed. (B) A growth curve was plotted by measuring the tumor volume. (C) Tumor weight was measured 30 days after the inoculation. (D) Representative images of Dec1 and Ki-67 immunostaining (magnification, x400) and apoptosis detection by TUNEL (magnification, x1,000) in U87-Dec1 and cherry tumors. (E) Box-whisker plot presenting the difference in proliferative index between U87-Dec1 and cherry tumors. (F) Box-whisker plot presenting the difference of apoptotic index between U87-Dec1 and cherry tumors. TMZ, temozolomide; Dec1, differentiated embryo chondrocyte expressed gene 1; TUNEL, terminal deoxynucleotidyl transferase dUTP nick end labeling.

gliomas. The present study provided additional evidence that high Dec1 expression negatively affected the response to TMZ chemotherapy in patients with HGG, which indicated that Decl was a prognostic factor and predictive factor for the response to TMZ chemotherapy in glioma patients. Given this evidence, high Decl expression may be correlated with poor prognosis in patients with glioma.

Traditionally, prognostic factors intend to objectively predict patient clinical outcome independent of treatment, while predictive factors aim to foretell the response of a patient to a specific therapeutic intervention and are associated with tumor sensitivity or resistance to the therapy. However, various factors exhibit both prognostic and predictive significance. For instance, human epidermal growth factor receptor 2 (HER2) has been validated as a prognostic factor and predictor of response to HER2-targeting therapy (41).

The original aim of the current study was to investigate the correlation of Dec1 expression with tumor malignancy and 
determine whether Decl expression may predict the outcome in 157 cases of newly diagnosed glioma. Surprisingly, the 86 cases of the TMZ-treated glioma patients were classified into resistant- and sensitive-groups according to the duration of PFS, a statistical significance between Dec1 expression levels and the response to TMZ chemotherapy was detected $(\mathrm{P}=0.020$; Table I). Furthermore, the low Dec1 expression group exhibited longer PFS and OS compared with the high Dec1 expression group in the TMZ-treated patients with HGG (Fig. 2D). Together this evidence revealed that, similar to HER2, Dec1 displayed a strong prognostic effect, and also appeared to predict a poor response to TMZ treatment.

Standard therapeutic strategies of maximal tumor resection and postoperative radiation combined with TMZ chemotherapy are almost never curative for GBMs, due to the infiltrative nature of GBMs, and the intrinsic or acquired resistance to radiation and chemotherapy (42). Therefore, identifying mechanisms of resistance to TMZ chemotherapy in GBMs may provide useful information for further individualized therapeutic strategies. The results of the current study indicated that Dec1 overexpression significantly attenuated the antiglioma effect of TMZ in vivo (Fig. 4). In addition, several previous studies provide evidence of the involvement of Dec1 in TMZ resistance. One report observed that Dec1 and Dec2 decreased mutL homolog 1 (MLH1) expression via binding to the E-box motifs in the MLH1 promoter region (43). MLH1 is considered to be an important factor in the DNA mismatch repair (MMR) pathway, and defects in this pathway are critical for mediating the tolerance of the cytotoxic effect of alkylating agents (44). Furthermore, the cytotoxicity of TMZ was low in colon cancer cells lines with hMLH1 mutation, and all five cell lines in the National Cancer Institute tumor panel that were deficient in hMLH1 activity were resistant to TMZ $(45,46)$. Therefore, as a MLH1 repressor, Dec1 overexpression may consequently decrease the cytotoxicity of TMZ. Additionally, it is well established that via the MMR system, TMZ induces double-strand breaks and triggers p53-dependent cell cycle arrest and apoptosis (47). As a novel target gene of the p53 family, Dec1 inhibited DNA damage-induced cell death by attenuating p53 induction of macrophage inhibitory cytokine-1. Furthermore, as a hypoxia-regulated transcription factor, Dec1 is upregulated by hypoxia in cancer, and the hypoxia-adaptation involves decreased leakage of reactive oxygen species (ROS) $(48,49)$. TMZ-induced DNA damage caused enhanced ROS generation and decreased ROS production-induced acquisition of chemoresistance (50). Generally, chemoresistance in gliomas may be influenced by the dysregulation of apoptosis-regulating genes and proteins. The results of the present study demonstrated an antagonizing role of high Decl expression in TMZ-induced apoptosis of the tumor cells in TMZ-treated recurrent GBMs, which was consistent with previous studies indicating that Dec1 is an anti-apoptotic transcription factor (12-15,31-33). Together this evidence suggests that Decl is involved in TMZ-resistance and suggests that inhibition of Dec1 expression may enhance the cytotoxicity of TMZ. The underlying mechanisms involved require further investigation.

In conclusion, the data of the present study provided evidence that Dec1 expression is closely associated with the malignant grade and TMZ-induced apoptosis of human glioma. It was additionally demonstrated that Dec1 is a prognostic indicator to evaluate the outcome of patients with glioma. Furthermore, Decl expression negatively affected the response to TMZ chemotherapy in patients with HGG. This valuable prognostic indicator for clinical outcome and predictive factor of the response to TMZ chemotherapy may be a potential novel molecular target for glioma biological therapy, particularly in patients with TMZ-resistance.

\section{Acknowledgements}

This study was supported by the National Natural Science Foundation of China (grant nos. 81001123 and 81572469) and the Natural Science Foundation of Shaanxi Province of China (grant no. S2012jc7293).

\section{References}

1. Ohgaki H: Epidemiology of brain tumors. Methods Mol Biol 472: 323-342, 2009.

2. Louis DN, Ohgaki H, Wiestler OD, Cavenee WK, Burger PC, Jouvet A, Scheithauer BW and Kleihues P: The 2007 WHO classification of tumours of the central nervous system. Acta Neuropathol 114: 97-109, 2007.

3. Schneider T, Mawrin C, Scherlach C, Skalej M and Firsching R: Gliomas in adults. Dtsch Arztebl Int 107: 799-807; quiz 808, 2010.

4. DeAngelis LM: Brain tumors. N Engl J Med 344: 114-123, 2001.

5. Chamberlain MC: Temozolomide: Therapeutic limitations in the treatment of adult high-grade gliomas. Expert Rev Neurother 10: 1537-1544, 2010.

6. Cancer Genome Atlas Research Network: Comprehensive genomic characterization defines human glioblastoma genes and core pathways. Nature 455: 1061-1068, 2008.

7. Shen M, Kawamoto T, Yan W, Nakamasu K, Tamagami M, Koyano Y, Noshiro M and Kato Y: Molecular characterization of the novel basic helix-loop-helix protein DEC1 expressed in differentiated human embryo chondrocytes. Biochem Biophys Res Commun 236: 294-298, 1997.

8. Boudjelal M, Taneja R, Matsubara S, Bouillet P, Dolle P and Chambon P: Overexpression of Stra13, a novel retinoic acid-inducible gene of the basic helix-loop-helix family, inhibits mesodermal and promotes neuronal differentiation of P19 cells. Genes Dev 11: 2052-2065, 1997.

9. Rossner MJ, Dörr J, Gass P, Schwab MH and Nave KA: SHARPs: Mammalian enhancer-of-split- and hairy-related proteins coupled to neuronal stimulation. Mol Cell Neurosci 10: 460-475, 1997.

10. Bhawal UK, Sato F, Arakawa Y, Fujimoto K, Kawamoto T, Tanimoto K, Ito Y, Sasahira T, Sakurai T, Kobayashi M, et al: Basic helix-loop-helix transcription factor DEC1 negatively regulates cyclin D1. J Pathol 224: 420-429, 2011.

11. Sun H and Taneja R: Stra13 expression is associated with growth arrest and represses transcription through histone deacetylase (HDAC)-dependent and HDAC-independent mechanisms. Proc Natl Acad Sci USA 97: 4058-4063, 2000.

12. Li Y, Zhang H, Xie M, Hu M, Ge S, Yang D, Wan Y and Yan B: Abundant expression of DEC1/stra13/sharp2 in colon carcinoma: Its antagonizing role in serum deprivation-induced apoptosis and selective inhibition of procaspase activation. Biochem J 367: 413-422, 2002.

13. Thin TH, Li L, Chung TK, Sun H and Taneja R: Stra13 is induced by genotoxic stress and regulates ionizing-radiation-induced apoptosis. Embo Rep 8: 401-407, 2007.

14. Ehata S, Hanyu A, Hayashi M, Aburatani H, Kato Y, Fujime M, Saitoh M, Miyazawa K, Imamura T and Miyazono K: Transforming growth factor-beta promotes survival of mammary carcinoma cells through induction of antiapoptotic transcription factor DEC1. Cancer Res 67: 9694-9703, 2007.

15. Li Y, Xie M, Yang J, Yang D, Deng R, Wan Y and Yan B: The expression of antiapoptotic protein survivin is transcriptionally upregulated by DEC1 primarily through multiple sp1 binding sites in the proximal promoter. Oncogene 25: 3296-3306, 2006. 
16. Seimiya M, Bahar R, Wang Y, Kawamura K, Tada Y, Okada S, Hatano M, Tokuhisa T, Saisho H, Watanabe T, et al: Clast5/Stra13 is a negative regulator of $\mathrm{B}$ lymphocyte activation. Biochem Biophys Res Commun 292: 121-127, 2002.

17. Sun H, Lu B, Li RQ, Flavell RA and Taneja R: Defective T cell activation and autoimmune disorder in Stra13-deficient mice. Nat Immunol 2: 1040-1047, 2001.

18. Miyazaki K, Miyazaki M, Guo Y, Yamasaki N, Kanno M, Honda $\mathrm{Z}$, Oda $\mathrm{H}$, Kawamoto $\mathrm{H}$ and Honda $\mathrm{H}$ : The role of the basic helix-loop-helix transcription factor Dec1 in the regulatory T cells. J Immunol 185: 7330-7339, 2010

19. Yun Z, Maecker HL, Johnson RS and Giaccia AJ: Inhibition of PPAR gamma 2 gene expression by the HIF-1-regulated gene DEC1/Stra13: A mechanism for regulation of adipogenesis by hypoxia. Dev Cell 2: 331-341, 2002.

20. Iizuka $\mathrm{K}$ and Horikawa Y: Regulation of lipogenesis via BHLHB2/DEC1 and ChREBP feedback looping. Biochem Biophys Res Commun 374: 95-100, 2008.

21. Yamada K, Ogata-Kawata H, Matsuura K and Miyamoto K SHARP-2/Stra13/DEC1 as a potential repressor of phosphoenolpyruvate carboxykinase gene expression. FEBS Lett 579 $1509-1514,2005$

22. Nakashima A, Kawamoto T, Honda KK, Ueshima T, Noshiro M, Iwata T, Fujimoto K, Kubo H, Honma S, Yorioka N, et al: DEC1 modulates the circadian phase of clock gene expression. Mol Cell Biol 28: 4080-4092, 2008.

23. Rossner MJ, Oster H, Wichert SP, Reinecke L, Wehr MC Reinecke J, Eichele G, Taneja R and Nave KA: Disturbed clockwork resetting in Sharp-1 and Sharp-2 single and double mutant mice. PLoS One 3: e2762, 2008.

24. Honma S, Kawamoto T, Takagi Y, Fujimoto K, Sato F, Noshiro M, Kato $\mathrm{Y}$ and Honma K: Dec1 and Dec2 are regulators of the mammalian molecular clock. Nature 419: 841-844, 2002.

25. Chakrabarti J, Turley H, Campo L, Han C, Harris AL, Gatter KC and Fox SB: The transcription factor DEC1 (stra13, SHARP2) is associated with the hypoxic response and high tumour grade in human breast cancers. Br J Cancer 91: 954-958, 2004

26. Zheng Y, Jia Y, Wang Y, Wang M, Li B, Shi X, Ma X, Xiao D and Sun Y: The hypoxia-regulated transcription factor DEC1 (Stra13, SHARP-2) and its expression in gastric cancer. OMICS 13: 301-306, 2009.

27. Ivanova AV, Ivanov SV, Danilkovitch-Miagkova A and Lerman MI Regulation of STRA13 by the von Hippel-Lindau tumor suppressor protein, hypoxia, and the UBC9/ubiquitin proteasome degradation pathway. J Biol Chem 276: 15306-15315, 2001.

28. Giatromanolaki A, Koukourakis MI, Sivridis E, Turley H, Wykoff CC, Gatter KC and Harris AL: DEC1 (STRA13) protein expression relates to hypoxia-inducible factor 1-alpha and carbonic anhydrase- 9 overexpression in non-small cell lung cancer. J Pathol 200: 222-228, 2003.

29. Zhang L and Li QQ: Embryo-chondrocyte expressed gene 1, downregulating hypoxia-inducible factor lalpha, is another marker of lung tumor hypoxia. Acta Pharmacol Sin 28: 549-558, 2007.

30. Shi XH, Zheng Y, Sun Q, Cui J, Liu QH, Qü F and Wang YS: DEC1 nuclear expression: A marker of differentiation grade in hepatocellular carcinoma. World J Gastroenterol 17: 2037-2043, 2011.

31. Qian Y, Zhang J, Yan B and Chen X: DEC1, a basic helix-loop-helix transcription factor and a novel target gene of the p53 family, mediates p53-dependent premature senescence. J Biol Chem 283: 2896-2905, 2008.

32. Qian Y,Jung YS and Chen X: Differentiated embryo-chondrocyte expressed gene 1 regulates p53-dependent cell survival versus cell death through macrophage inhibitory cytokine-1. Proc Natl Acad Sci USA 109: 11300-11305, 2012.

33. Sato F, Bhawal UK, Kawamoto T, Fujimoto K, Imaizumi T, Imanaka T, Kondo J, Koyanagi S, Noshiro M, Yoshida $\mathrm{H}$, et al: Basic-helix-loop-helix (bHLH) transcription factor DEC2 negatively regulates vascular endothelial growth factor expression. Genes Cells 13: 131-144, 2008.

34. Ivanov SV, Salnikow K, Ivanova AV, Bai L and Lerman MI: Hypoxic repression of STAT1 and its downstream genes by a pVHL/HIF-1 target DEC1/STRA13. Oncogene 26: 802-812, 2007.
35. Wykoff CC, Pugh CW, Maxwell PH, Harris AL and Ratcliffe PJ: Identification of novel hypoxia dependent and independent target genes of the von Hippel-Lindau (VHL) tumour suppressor by mRNA differential expression profiling. Oncogene 19: 6297-6305, 2000

36. Ivanova AV, Ivanov SV, Zhang X, Ivanov VN, Timofeeva OA and Lerman MI: STRA13 interacts with STAT3 and modulates transcription of STAT3-dependent targets. J Mol Biol 340: 641-653, 2004.

37. Wang W, Reiser-Erkan C, Michalski CW, Raggi MC, Quan L, Yupei Z, Friess H, Erkan M and Kleeff J: Hypoxia inducible BHLHB2 is a novel and independent prognostic marker in pancreatic ductal adenocarcinoma. Biochem Biophys Res Commun 401: 422-428, 2010

38. Meyer SE, Hasenstein JR, Baktula A, Velu CS, Xu Y, Wan H, Whitsett JA, Gilks CB and Grimes HL: Kruppel-like factor 5 is not required for K-RasG12D lung tumorigenesis, but represses ABCG2 expression and is associated with better disease-specific survival. Am J Pathol 177: 1503-1513, 2010.

39. Soon MS, Hsu LS, Chen CJ, Chu PY, Liou JH, Lin SH, Hsu JD and Yeh KT: Expression of Krüppel-like factor 5 in gastric cancer and its clinical correlation in Taiwan. Virchows Arch 459: 161-166, 2011.

40. Stupp R, Hegi ME, Mason WP, van den Bent MJ, Taphoorn MJ, Janzer RC, Ludwin SK, Allgeier A, Fisher B, Belanger K, et al: Effects of radiotherapy with concomitant and adjuvant temozolomide versus radiotherapy alone on survival in glioblastoma in a randomised phase III study: 5 -year analysis of the EORTC-NCIC trial. Lancet Oncol 10: 459-466, 2009.

41. Pazo CR and Antón A: Advanced HER2-positive gastric cancer: Current and future targeted therapies. Crit Rev Oncol Hematol 85: 350-362, 2013.

42. Stupp R, Mason WP, van den Bent MJ, Weller M, Fisher B, Taphoorn MJ, Belanger K, Brandes AA, Marosi C, Bogdahn U, et al: Radiotherapy plus concomitant and adjuvant temozolomide for glioblastoma. N Engl J Med 352: 987-996, 2005.

43. Nakamura H, Tanimoto K, Hiyama K, Yunokawa M, Kawamoto T, Kato Y, Yoshiga K, Poellinger L, Hiyama E and Nishiyama M: Human mismatch repair gene, MLH1, is transcriptionally repressed by the hypoxia-inducible transcription factors, DEC1 and DEC2. Oncogene 27: 4200-4209, 2008.

44. Sarkaria JN, Kitange GJ, James CD, Plummer R, Calvert H, Weller M and Wick W: Mechanisms of chemoresistance to alkylating agents in malignant glioma. Clin Cancer Res 14: 2900-2908, 2008.

45. Taverna P, Liu L, Hanson AJ, Monks A and Gerson SL: Characterization of MLH1 and MSH2 DNA mismatch repair proteins in cell lines of the NCI anticancer drug screen. Cancer Chemother Pharmacol 46: 507-516, 2000.

46. Liu L, Markowitz S and Gerson SL: Mismatch repair mutations override alkyltransferase in conferring resistance to temozolomide but not to 1,3-bis (2-chloroethyl) nitrosourea. Cancer Res 56: 5375-5379, 1996.

47. Hickman MJ and Samson LD: Role of DNA mismatch repair and p53 in signaling induction of apoptosis by alkylating agents. Proc Natl Acad Sci USA 96: 10764-10769, 1999.

48. Turley H, Wykoff CC, Troup S, Watson PH, Gatter KC and Harris AL: The hypoxia-regulated transcription factor DEC1 (Stra13, SHARP-2) and its expression in human tissues and tumours. J Pathol 203: 808-813, 2004

49. Ali SS, Hsiao M, Zhao HW, Dugan LL, Haddad GG and Zhou D: Hypoxia-adaptation involves mitochondrial metabolic depression and decreased ROS leakage. PLoS One 7: e36801, 2012.

50. Oliva CR, Moellering DR, Gillespie GY and Griguer CE: Acquisition of chemoresistance in gliomas is associated with increased mitochondrial coupling and decreased ROS production. PLoS One 6: e24665, 2011 . 